\title{
Right Testis
}

National Cancer Institute

\section{Source}

National Cancer Institute. Right Testis. NCI Thesaurus. Code C49326.

The testis on the right side of the body. 